\title{
Variations
}

Variations

Revue internationale de théorie critique

$19 \mid 2016$

Critique des humanités numériques

\section{Crises du capitalisme, migrations et bureaucratie européenne}

Entretien avec Nina Živančević

David Graeber et Nina Živančević

Traducteur : Christophe Magis

\section{(2) OpenEdition}

Journals

Édition électronique

URL : http://journals.openedition.org/variations/760

DOI : 10.4000/variations. 760

ISSN : 1968-3960

Éditeur

Les amis de Variations

Référence électronique

David Graeber et Nina Živančević, "Crises du capitalisme, migrations et bureaucratie européenne », Variations [En ligne], 19 | 2016, mis en ligne le 06 avril 2016, consulté le 22 avril 2019. URL : http:// journals.openedition.org/variations/760 ; DOI : 10.4000/variations.760

Ce document a été généré automatiquement le 22 avril 2019

Les ami•e•s de Variations 


\title{
Crises du capitalisme, migrations et bureaucratie européenne
}

\author{
Entretien avec Nina Živančević
}

David Graeber et Nina Živančević

Traduction : Christophe Magis

\section{NOTE DE L'ÉDITEUR}

Éminent anthropologue et militant anarchiste américain, David Graeber est l'un des intellectuels les plus influents du monde anglophone selon le New York Times. Il est professeur d'anthropologie à la London School of Economics et l'un des fondateurs du mouvement américain de protestation «Occupy Wall Street » ainsi que l'auteur du slogan « We are the 99\% ». Auteur du best-seller Dette : 5000 ans d'histoire (Debt : the First 5000 years) préfacé par Thomas Piketty, ainsi que de nombreux autres textes, son livre le plus récent s'intitule Bureaucratie (Utopia of Rules, about Technology, Stupidity and Hidden Pleasures of Bureaucracy). Dans cet entretien, conduit par Nina Živančević et traduit et adapté pour la revue par Christophe Magis, Graeber débat des causes et contextes de l'immigration, de la situation de détresse des réfugiés syriens et ses effets catastrophiques sur l'Europe de l'Est, ainsi que de la prétendue « crise européenne ».

NINA ŽıVANČEvić : Nous sommes tous préoccupés par la crise européenne, par les migrants et réfugiés, pas seulement en Grèce mais aussi en "Mitteleuropa » ou dans l'Europe de l'Est. Considères-tu ces différentes manifestations de la crise comme des éléments isolés et différents ou les vois-tu comme des événements intrinsèquement connectés?

David Graeber : Vois-tu, je considérerais tous ces éléments dans un processus continu : le développement de l'Empire, l'Empire dans sa chute et sa désintégration. Ce processus pourrait être celui qui suit la période de crise de la dette, qui a commencé dans les pays du Tiers-Monde et s'est déplacé jusqu'à atteindre le sud de l'Europe, en direction du centre de l'Empire. Ce processus a été rendu possible grâce au travail des forces de la 
Résistance. Par conséquent, on le retrouve aussi dans les régions de l'Asie et de l'Amérique latine, ce qui signifie que nous parlons d'un processus général d'implosion. Parce que l'Empire américain est en train de s'effondrer mais qu'on n'a pas encore trouvé de quoi le remplacer. Alors, des vagues de migrants arrivent en Europe, mais l'Europe est dans une profonde dépression économique... Au total, je dirais que tous ces éléments sont, tous, des manifestations d'un certain processus historique.

Nz: Nous quittons en ce moment ce que Foucault a appelé «la société disciplinaire » pour entrer dans la «société de contrôle » où chacun surveille tout le monde. Comment cette nouvelle mentalité sociale influence-t-elle nos attitudes envers les réfugiés et migrants, ici en Europe?

DG : Je me rappelle, enfant, avoir lu l'histoire de la Grèce ancienne: il y avait des milliers de personnes qui faisaient leurs valises et se déplaçaient d'un endroit du monde vers un autre. Ils allaient vers l'Asie, par exemple. Et là, j'ai compris que quelque activité «illégale » était en train de se produire alors, dans une forme différente de celle qui se produit aujourd'hui. Nous sommes témoins d'un contrôle global incroyable des frontières terrestres et des lois qui régissent ces frontières. Alors qu'on sait que la technologie a beaucoup progressé, nous sommes toujours confrontés à une quantité incroyable de murs et de frontières qui nous sont imposés. Qu'ils soient virtuels ou non ces murs découpent notre planète en des millions de morceaux séparés. Jusqu'à il y a peu, c'était pour nous une sorte d'abstraction, mais c'est désormais devenu notre réalité physique.

NZ : Comment se fait-il que nous revenions toujours à la notion de «mur »?

DG : Peut-être que nous avons besoin de ces murs après tout ! Puisque nous avons parlé de Foucault et du modèle spécifique du panoptique, de la surveillance de tout le monde, il faut souligner une chose qu'en général, les gens oublient à ce propos : c'est que ce panoptique est aussi une prison que les prisonniers pourraient quitter, si seulement elle n'avait pas de murs ni de gardiens susceptibles de devenir violents en cas de tentative d'échappée. En d'autres termes, nous avons toujours en tête une notion de la violence métaphysique qui permet la manifestation de toutes les autres formes de violence. Ce qui est incroyable ici, c'est qu'il existe un réseau extrêmement puissant de l'information bureaucratique nécessaire pour que les individus puissent se déplacer d'un endroit du monde à un autre. Imagine par exemple que je sois réfugié syrien et que, dans l'urgence de la fuite de mon pays, je n'ai pas emporté avec moi mon certificat de naissance ! Si je ne l'ai pas, qu'est-ce que je fais? J'invente toute ma propre histoire? Et si je veux contacter ma famille en Syrie afin de recevoir mon certificat, récupérer mes affaires, etc. alors, je vois comme le pouvoir de ces «murs" agit: où que je sois dans le monde, j'appartiens à mon pays, à cette partie du monde qui est derrière le mur. Aujourd'hui, on ne peut même plus imaginer à quoi ressemblait une situation comme il y a deux mille ans, lorsque les gens pouvaient se déplacer vers quelque endroit qu'ils voulaient, autant qu'ils le souhaitaient.

NZ : Tu veux dire lors de l'ère «pré-Paléolithique»?

DG : Non, pas vraiment. Mais à l'époque du féodalisme, les gens voyageaient d'Afrique en Angleterre, par exemple. Et je ne parle pas ici des esclaves! Ces travailleurs n'étaient pas des esclaves, ils pouvaient simplement entrer physiquement dans un autre pays et personne ne les en empêchait, dans aucun pays. Et ce qui est intéressant, c'est qu'ils étaient accueillis chaleureusement par les habitants. La notion d'hospitalité était très développée et l'hôte ressentait comme une obligation morale d'accueillir un étranger : 
il lui servait les meilleurs repas à sa table pendant trois jours. Bon, peut-être, si l'invité restait un peu trop longtemps, son hôte pouvait en faire un esclave - mais nous n'entrerons pas ici dans les aspects et raisons psychologiques expliquant l'hospitalité de quelqu'un (rires). L'anthropologue autrichien Franz Steiner a cependant beaucoup écrit à ce propos. Il a rédigé une thèse de doctorat « Sur la nature de l'esclavage » qu'il a perdu par la suite dans le train lors de son voyage de Cambridge à Londres et il est mort peu de temps après. Toutefois, il a développé cette thèse en rapport avec son expérience personnelle: lui-même était un réfugié en Angleterre tout en étant en même temps un éminent professeur. Ainsi, il a toujours dit qu'on l'invitait volontiers à dormir, qu'on lui servait à boire et à manger, qu'on lui demandait à lire son travail intéressant, etc. ...mais seulement pour lui demander à la fin toutes sortes de faveurs comme par exemple de faire la vaisselle.

Nz : Cet été, j'ai été témoin d'un afflux incroyable de réfugiés venant vers Belgrade. Ils étaient tous accueillis humainement, mais je voudrais savoir maintenant comment les gouvernements des pays de l'Europe de l'Ouest peuvent s'imaginer un instant que l'on prendra soin des réfugiés dans les petits pays comme la Slovénie, la Serbie, la Roumanie lorsque ces pays plus gros et plus riches refusent de les aider.

DG : Il semblerait que dans ce qu'on appelle l'« Europe de l'Ouest », les administrations et gouvernements ont commencé à calculer combien de réfugiés ils pouvaient accepter, quels boulots ils pouvaient occuper et où est-ce qu'ils se sentiraient le mieux. Assez étrangement, ils sont arrivés à la conclusion, d'après ces calculs, que le meilleur pays pour les réfugiés était la Hongrie...

$\mathbf{N Z}$ : Cela me mène à ma question suivante : est-ce que cet afflux de réfugiés encourage les partis nationalistes dans leur affolement par rapport aux cadres généraux des politiques des pays d'Europe de l'Ouest?

DG : Oui, c'est une résultante dans certains pays! Mais il me semble que les partis nationalistes et leurs politiques anti-immigration sont aujourd'hui surtout présents dans les petites villes et villages où les réfugiés ne finiront pas de toute façon. En France, par exemple et dans d'autres pays où un « Front National » est actif, les cadres de tels partis vivent dans ces lieux qui échappent à l'afflux des migrants. À Londres, par exemple, les représentants du Front National n'ont jamais rencontré un immigré en personne : ils vivent dans des banlieues huppées où les migrants ne vont jamais. Et s'ils devaient en rencontrer, ils ne sauraient pas même quoi dire, que demander, comment interagir avec eux.

NZ : Parlons d'autre chose : Jacques Rancière a dit que la politique et l'art entretiennent une relation fictive, qu'en penses-tu?

DG : Je suis d'accord sur le fait qu'il y a une relation imaginaire et fictive entre eux. Tu sais, les individus projettent toutes sortes de désirs sur les étrangers lorsqu'ils les rencontrent! Des désirs pervers et étranges qui les font toujours trouver les étrangers trop différents d'eux. Pourtant, nous savons qu'une pré-condition pour construire une solidarité avec les réfugiés est le sentiment d'égalité et d'être-ensemble. Il y a un autre élément : les gens aiment bien voir les réfugiés comme des victimes. Ils éprouvent de la sympathie et de l'empathie pour eux jusqu'à un certain point, en s'imaginant euxmêmes dans une situation similaire. Tout cela, jusqu'au moment où ces "pauvres " réfugiés commencent à se sentir bien. Alors, les sentiments de leurs hôtes changent, ils commencent à avoir certains doutes quant à ces réfugiés. La question est : comment combattre ce sentiment? 
Nz : Est-ce le sentiment qui émerge lors de la rencontre avec l'Autre, comme dirait Levinas? Aie peur lorsque tu vois un étranger, qui qu'il soit?

DG : La notion de l'Autre n'implique pas uniquement la peur mais également le sentiment d'attraction envers l'Autre. Lorsque j'ai lu les observations de Levinas sur le visage humain, je les ai comprises de manière paradoxale - puisque nous sommes tous les mêmes, dans le sens où nous sommes tous des êtres humains - comme indiquant que nous sommes tous différents. J'y ai pensé beaucoup lorsque je vivais à Madagascar, dans une culture différente de la mienne. Ces visages ne sont pas seulement différents des nôtres, ils diffèrent entre eux, tout comme nous sommes différents les uns des autres, même lorsque nous sommes de la même culture. En ce sens, Levinas cherche à montrer l'unicité absolue de chacun d'entre nous, de chaque visage que nous ne pouvons jamais entièrement intégrer, ce qui peut être douloureux mais renforce cette conscience en nous que chacun est unique et spécial. Et que cela construit une partie de ce qui nous rend humains, cette sorte d'appropriation constante de la conscience que chaque être humain est unique. En tant qu'anthropologue, je suis constamment en train d'étudier l'Autre et me rends compte en permanence du fait que l'Autre est différent et que nous sommes toujours enclins à ne pas comprendre l'Autre ou sa culture - nous touchons là un point de limitation en nous : il est fréquent que je ne comprenne pas mon propre frère qui est un grand inconnu pour moi ! À Madagascar, j'ai rencontré quelqu'un qui ressemblait tellement à mon ex-compagne que je ne savais pas comment m'adresser à cette personne! Pourtant, nous différons tellement qu'au final, nous arrivons à la conclusion que les gens sont tous les mêmes de plein de façons différentes.

Nz: Les sociologues européens disent que les réfugiés et les immigrants ne sont pas un réel fardeau pour l'Europe en ce qu'ils peuvent être providentiels dans une Europe qui manque de main d'œuvre et qui a besoin d'une plus importante force de travail, qui ne serait ni physique ni intellectuelle mais de ce que Toni Negri nomme «la force du travail immatériel ».

DG : Oui, je vois. Je vais faire un parallèle avec ce qui se passe aux Etats-Unis : en Amérique du Nord, la situation est claire : jusqu'à il y a peu, tous les immigrants sans papiers avaient un statut de parias : ils n'avaient ni droit de vote ni aucun autre droit. Ils n'avaient évidemment pas de syndicat pour les représenter. Il n'y a que récemment que s'est formé un mouvement pour les droits civils permettant à ces individus de s'exprimer. En même temps, l'immigration depuis le Mexique devient proéminente aux USA et les politiciens se demandent toujours s'ils doivent expulser ces immigrants du pays ou pas - ils ont du mal à s'entendre sur ce problème. Et ce que représentent ces immigrants aujourd'hui, c'est exactement le type de travailleurs que la classe capitaliste a toujours recherché : un groupe d'individus qui n'ont aucun droit légal et qui ne sont pas défendus en tant que travailleurs par aucun syndicat. Comme ils n'ont pas le droit de se syndiquer, ces travailleurs sont dans un flou légal. On parle donc ici de travail qui est en réalité hors-la-loi. Ces travailleurs illégaux n'ont pas de droits et peuvent à tout moment être jetés selon le bon vouloir du capitaliste.

NZ : Et comment cet afflux de nouveaux migrants peut-il changer le statut du travail en Europe aujourd'hui ? Y a-t-il un réel danger pour ceux qui travaillent en Europe de perdre plus facilement leur travail à mesure qu'un plus grand nombre de migrants sera en mesure de les remplacer?

DG : Je ne vois pas là un réel danger car n'oublions pas que cet afflux massif de réfugiés crée également un certain nombre d'emplois sociaux : il y a de nouveaux besoins, pour de nouveaux services aux populations qui peuvent être satisfaits par le travail des 
migrants. En ce sens, si le nombre d'habitants double, le besoin pour l'approvisionnement en nourriture et les emplois dans ce domaine d'activités doublent également, comme les services assurant la survie quotidienne. Dans l'histoire, nous avons été témoins de peurs analogues à celle que tu mentionnes, par exemple, que si les Saxons arrivaient, ils allaient exterminer les Celtes etc. En réalité, ce qui s'est passé, c'est que leurs territoires se sont intégrés et qu'on les a tous appelés Saxons à partir de là.

NZ : Es-tu d'accord avec la théorie habermassienne selon laquelle le « Problème européen » découle d'un certain manque de cohésion et de collaboration entre les pays membres de I'Union Européenne, alors que ces derniers sont écrasés par un fonctionnement extrêmement bureaucratique? Et que le système monétaire européen a été si affaibli par les pressions que cette bureaucratie produit et exerce qu'il ne peut, en conséquence, pas réagir de manière adéquate dans un moment de crise financière extrême? Tu mentionnes ce problème dans ton nouveau livre The Utopia of Rules. On Technology, Stupidity and Hidden Pleasures of Bureaucracy.

DG : Oui, nous y assistons en ce moment même! Nous assistons à un élan vers une bureaucratisation extrême de chaque aspect de notre réalité quotidienne. Je n'ai pas les chiffres exacts, personne n'a vraiment cherché à compter mais nous devrions prêter attention à la quantité incroyable de temps qu'une personne ordinaire passe aujourd'hui, dans tout pays européen à remplir des formulaires! Il semble que les statistiques nous informent que nous passons tous environ deux jours entiers par an à s'occuper de paperasserie! Ce qui est effrayant en ce moment est à ce propos la fusion totale des sphères publiques et privée dans la bureaucratie. Hier encore, j'ai passé environ une heure à parler à ma banque. Comme d'habitude dans ce cas, c'est toujours avec une personne dont le nom est difficile à obtenir, qui est invisible et hors d'atteinte - c'est une approche bureaucratique classique du problème. Et si jamais tu réussis à atteindre cette personne, elle te dira que les lois qui la protègent (c'est à dire, qui protègent la banque) sont des lois formulées par l'État, ce qui n'est pas vrai car les « lois » régissant n'importe quelle banque sont formulées par le lobby banquier. Il faut alors se poser la question : est-ce le domaine de la sphère publique ou privée ? On voit bien qu'il y a eu une fusion totale entre les sociétés bancaires et l'État, le capitalisme tardif réalisant ses profits en contrôlant la sphère financière, notamment sur les individus endettés et avec des dettes qui sont créées au travers de processus bureaucratiques spécifiques.

On parle ici d'une certaine forme d'utopie car il est utopique de croire que les individus devraient toujours se comporter d'une certaine manière et que, sinon, ils seront punis par l'État. Les grandes entreprises créent les lois qui s'imposent à nous sous peine de punition si on ne les respecte pas. Ce mécanisme relève de la pure utopie et d'une sorte de bureaucratisation qui dévore tout sur son passage. Ce processus n'admet aucune flexibilité bien au contraire, il est profondément conservateur. Si Habermas a pris cela en considération, il a probablement réfléchi au développement technologique qui accompagne cette forme de capitalisme, avec tous les changements additionnels, les avancées scientifiques et les nouvelles formes d'utilisation de l'énergie. Nous avons également Internet qui est une sorte de bibliothèque géante. Si on l'avait montré à quelqu'un vivant dans les années 1950, cette personne se serait enthousiasmée: "Internet, c'est fantastique, c'est le tout dernier mot de la science ", sans penser une seconde à la possibilité que cette découverte scientifique puisse également tuer la créativité. On en est aussi arrivé là avec la bureaucratisation de l'éducation. 
Nz : Quel sujet t'intéresse particulièrement en ce moment? À propos de quoi aimerais-tu écrire?

DG : Je travaille avec un archéologue célèbre sur un livre dont le titre sera « the PirateKings of Madagascar ». Il faut savoir que la dernière fois qu'anthropologues et archéologues ont travaillé ensemble et fait de vraies découvertes, c'était à la fin des années 1960. Ils ont découvert à quel point on pouvait se faire des idées erronées sur l'histoire, comme celle selon laquelle dans le passé, les gens auraient vécu en petits groupes de trente à quarante personnes, ou dans des igloos par exemple, puis que l'âge de l'agriculture est arrivé, etc. puis qu'ensuite nous arrivons à la formation des villes, nous avons une classe dirigeante qui se forme, un état et tout le package civilisationnel qui va avec! Bon, le seul problème, c'est que rien de tout cela n'est vrai. Les trois premières civilisations que l'on connaît sont les suivantes : la civilisation Uruk (entre -4000 et -3200), la civilisation de la vallée de l'Indus et la civilisation mésopotamienne. Ce qui est intéressant, c'est que ces civilisations étaient structurées de manière égalitaire, ce qui, depuis notre perspective, nous paraît étrange voire impossible. À chaque fois, lorsqu'on est en présence d'un petit groupe d'individus qui se structure et s'organise, nous partons du principe que certains individus vont essayer de prendre le pouvoir sur les autres, mais dans ces cités anciennes, les citoyens avaient un statut égal. Par exemple, les bâtiments les plus grands n'étaient pas des palais ou des temples mais des bâtiments de santé, des bains publics auxquels de larges portions de la population pouvaient avoir accès. Les archéologues ont toujours pensé qu'il y avait un certain codex dans de telles cités, garantissant l'exercice du pouvoir ou la direction des affaires à un groupe spécial, mais il ne semble pas que cela se passait ainsi. Dans ces civilisations, si le système familial n'était peut-être pas très égalitaire, il apparaît que tout le reste l'était. C'est ce que je viens récemment de découvrir et je viens de commencer à écrire un livre à ce propos.

Par ailleurs, je poursuis l'écriture de mon livre sur les «bullshit jobs » en remontant jusqu'aux années 1930 de John Maynard Keynes. Ce dernier espérait que l'avancement technologique aiderait les gens à travailler moins ou à travailler le nombre d'heures de leur choix. Or, ce qui est arrivé, c'est qu'avec le développement technologique, les boulots de type bureaucratique ou de secrétariat n'ont fait que foisonner et se développer. Et si on demandait à cette armée de travailleurs «qu'avez vous fait aujourd'hui au travail ?", ils répondraient «oh, en gros rien, j'ai juste fixé mon écran toute la journée - mais ne le dites pas à mon patron!». Et en fait, tous ces gens qui sont dans le marketing, dans la promotion ainsi que les avocats des grandes sociétés, s'ils devaient soudainement disparaître de la surface de la Terre, nous ne remarquerions pas même leur absence. Et je n'entre même pas dans les aspects psychologiques du problème, à savoir ce qui se passe vraiment dans l'esprit de ces pauvres gens qui travaillent supposément sur quelque chose mais ne verront jamais le produit final de leur travail. Même si tu dois nettoyer le caniveau, certes, c'est un boulot dégoûtant mais bon, quelqu'un doit bien le faire et tu sais au moins concrètement en quoi consiste le travail. Maintenant, si tu penses à quelqu'un qui est directeur marketing pour la côte est des Etats-Unis dans une société qui s'appelle "Global Vision » : tu vois un pauvre mec qui voulait peut-être devenir musicien mais qui a eu un gosse et a dû trouver un "emploi stable ». Et le voilà avocat dans une société où il doit écrire un mémorandum par jour que personne ne lit! Je crois que Dostoïevski a bien décrit ce moment stupide dans une prison où les prisonniers 
devaient vider des boites de sable et ensuite les re-remplir... avec du sable - pour eux l'action la plus stupide et absurde - : voilà ce que doivent ressentir les gens qui travaillent dans ces bullshit jobs.

\section{AUTEURS}

\section{DAVID GRAEBER}

Professeur d'anthropologie à la London School of Economics 